\title{
NUMERICAL STUDY OF THE SYSTEMATIC ERROR IN MONTE CARLO SCHEMES FOR SEMICONDUCTORS
}

\author{
Orazio Muscato $^{1}$, Wolfgang Wagner ${ }^{2}$ and Vincenza Di Stefano ${ }^{3}$
}

\begin{abstract}
The paper studies the convergence behavior of Monte Carlo schemes for semiconductors. A detailed analysis of the systematic error with respect to numerical parameters is performed. Different sources of systematic error are pointed out and illustrated in a spatially one-dimensional test case. The error with respect to the number of simulation particles occurs during the calculation of the internal electric field. The time step error, which is related to the splitting of transport and electric field calculations, vanishes sufficiently fast. The error due to the approximation of the trajectories of particles depends on the ODE solver used in the algorithm. It is negligible compared to the other sources of time step error, when a second order Runge-Kutta solver is used. The error related to the approximate scattering mechanism is the most significant source of error with respect to the time step.
\end{abstract}

Mathematics Subject Classification. 82D37, $65 \mathrm{C} 05$.

Received July 30, 2008. Revised April 6, 2009.

Published online August 26, 2010.

\section{INTRODUCTION}

The combination of a statistical solution of the Boltzmann transport equation and a self-consistent solution of the Poisson equation continues to gain popularity as a viable approach to submicrometric device simulation $[5,7,16]$. In contrast to other simulation tools, like drift-diffusion or hydrodynamic models [1], which involve the solution of coupled systems of partial differential equations, the Boltzmann equation is treated by replacing the distribution function with a representative set of particles. The Monte Carlo approach is a useful tool, since it permits particular physical simulations unattainable in experiments, or even investigations of nonexistent materials in order to emphasize special features of the phenomenon under study. It provides an accurate description of carrier transport phenomena because various scattering mechanisms and band structure models are taken into account.

The Monte Carlo results are affected by discretization and stochastic errors, which influence their accuracy and efficiency. A study of the time step truncation in the bulk case has been performed in [10]. The aim of this paper is to study the discretization error in a prototype device, the $n^{+}-n-n^{+}$silicon diode. In Section 2

\footnotetext{
Keywords and phrases. Boltzmann-Poisson equations, electronic devices, Monte Carlo simulations.

1 Dipartimento di Matematica e Informatica, Università degli Studi di Catania, Viale Andrea Doria 6, 95125 Catania, Italy. muscato@dmi.unict.it

${ }^{2}$ Weierstrass Institute for Applied Analysis and Stochastics, Mohrenstrasse 39, 10117 Berlin, Germany. wagner@wias-berlin . de

3 Dipartimento di Matematica, Università degli Studi di Messina, Contrada Papardo 31, 98166 Messina, Italy.

vdistefano@dmi.unict.it
} 
we introduce the basic equations governing the transport phenomena as well as the main scattering mechanisms for silicon. In Section 3 the Monte Carlo algorithms are described. In particular, the self scattering technique and the constant time technique are recalled. In Section 4 the setup of the numerical experiments is defined. The main results are presented in Section 5. The systematic error due to the particle number and to the splitting time step is analyzed. Various sources of the discretization error are pointed out. Issues related to the efficiency of the algorithms are discussed. Finally, some conclusions are drawn in Section 6.

\section{BAsic EQUATIONS}

We study Monte Carlo algorithms (cf. [5]) for the Boltzmann transport equation of the form

$$
\left[\frac{\partial}{\partial t}+v(k) \cdot \nabla_{x}-\frac{q}{\hbar} E(t, x) \cdot \nabla_{k}\right] f(t, x, k)=(Q f)(t, x, k) .
$$

The solution $f(t, x, k)$ represents the probability density of finding an electron at time $t$ in the position $x$, with the wave-vector $k$.

In the quasi parabolic approximation the kinetic energy $\varepsilon(k)$ of an electron satisfies the relation

$$
\varepsilon(k)[1+\alpha \varepsilon(k)]=\frac{\hbar^{2}|k|^{2}}{2 m^{\star}}, \quad k \in \Omega,
$$

and the electron (group) velocity is given by

$$
v(k)=\frac{1}{\hbar} \nabla_{k} \varepsilon(k)=\frac{\hbar k}{m^{\star}[1+2 \alpha \varepsilon(k)]} .
$$

The effective electron mass $m^{\star}$ equals $0.32 m_{e}$ in silicon, the nonparabolicity factor $\alpha$ equals $0.5 \mathrm{eV}^{-1}$ in silicon, and $\hbar$ denotes Planck's constant divided by $2 \pi$. The domain $\Omega$ is called first Brillouin zone, which is a characteristic of each material. We refer to [5], Section 2.2.5, for further details.

The electric field is defined as

$$
E(t, x)=-\nabla_{x} \Phi(t, x)
$$

The electric potential $\Phi$ is related to the solution $f$ by the Poisson equation

$$
\epsilon \Delta_{x} \Phi(t, x)=q\left[n(t, x)-N_{D}(x)\right]
$$

where the electron density is given by

$$
n(t, x)=\int_{\Omega} f(t, x, k) \mathrm{d} k
$$

Here $N_{D}$ denotes the donor density, $q$ is the absolute value of the electron charge and $\epsilon$ is the permittivity.

The linear scattering collision operator has the form

$$
(Q f)(t, x, k)=\int_{\Omega} S\left(k^{\prime}, k\right) f\left(t, x, k^{\prime}\right) \mathrm{d} k^{\prime}-\lambda(k) f(t, x, k),
$$

where

$$
\lambda(k)=\int_{\Omega} S\left(k, k^{\prime}\right) \mathrm{d} k^{\prime}
$$

is the total scattering rate. 
Remark 2.1. The electron-electron interaction is taken into account in the framework of the mean field approximation through the Poisson equation (2.5). This is justified in the case of low electron density, for which the short range collisions between electrons can be neglected.

The main scattering mechanisms in silicon, at room temperature, are due to electron-phonon interactions (acoustic and optical). The transition rate from a state $k$ to a state $k^{\prime}$ is modeled as (cf. [6], Sect. III.D.1)

$$
S\left(k, k^{\prime}\right)=K_{0} \delta\left(\varepsilon\left(k^{\prime}\right)-\varepsilon(k)\right)+\sum_{i=1}^{6} K_{i}\left[\delta\left(\varepsilon\left(k^{\prime}\right)-\varepsilon(k)+\hbar \omega_{i}\right)\left(\mathrm{n}_{q_{i}}+1\right)+\delta\left(\varepsilon\left(k^{\prime}\right)-\varepsilon(k)-\hbar \omega_{i}\right) \mathrm{n}_{q_{i}}\right],
$$

where $\hbar \omega_{i}$ is a phonon energy. According to Bose-Einstein statistics, the phonon equilibrium distribution is given by

$$
\mathrm{n}_{q_{i}}=\frac{1}{\exp \left(\hbar \omega_{i} / k_{B} T_{L}\right)-1},
$$

where $T_{L}$ is the lattice temperature. The function

$$
K_{0}=\frac{k_{B} T_{L} \Xi_{d}^{2}}{4 \pi^{2} \hbar \rho v_{s}^{2}}
$$

represents the intravalley elastic scattering transition rate, where $\Xi_{d}$ is the acoustic-phonon deformation potential, $\rho$ is the silicon mass density and $v_{s}$ denotes the sound velocity of the longitudinal acoustic mode. The inelastic scattering rates have the form

$$
K_{i}=\frac{Z_{f}\left(D_{t} K_{i}\right)^{2}}{8 \pi^{2} \rho \omega_{i}}, \quad i=1, \ldots, 6,
$$

where $D_{t} K_{i}$ is the deformation potential for the $i$-th optical phonon, and $Z_{f}$ is the number of final equivalent valleys for the considered inter-valley scattering.

Finally, boundary conditions are added to (2.5), taking into account an external field. Boundary conditions to (2.1) are more complicated. In the one-dimensional case considered later we will assume periodic boundaries.

\section{Monte Carlo algorithms}

The Monte Carlo approach is based on a stochastic particle system of the form

$$
\left(x_{i}(t), k_{i}(t)\right), \quad i=1, \ldots, N, \quad t \geq 0,
$$

which depends on a time step $\Delta t$. The system (3.1) provides a numerical algorithm for equation (2.1) in the sense that functionals of the solution $f$ are approximated (as $N \rightarrow \infty$ and $\Delta t \rightarrow 0$ ) by averages over the particle system.

\subsection{Ingredients of the time evolution}

The stochastic particles mimic the evolution of electrons in time and space inside the device. Their behavior is determined by the action of external and self-consistent electric fields and of the given scattering mechanisms.

\section{Field calculation}

During time step $\Delta t$, the electric field (2.4) is kept fixed $(=\tilde{E}(x))$ and the particles are moved and scattered independently of each other. At the end of the time step, the electric field is recalculated according to the density provided by the system. This splitting procedure tackles the nonlinearity of the system of equations (2.1)-(2.6), which is in the electric field depending on the solution (cf. Rem. 2.1). 


\section{Free flight}

During the free flight, particles move according to Newton's equations of motion (cf. (2.3))

$$
\frac{\mathrm{d}}{\mathrm{d} t} x_{i}(t)=v\left(k_{i}(t)\right), \quad \frac{\mathrm{d}}{\mathrm{d} t} k_{i}(t)=-\frac{q}{\hbar} \tilde{E}\left(x_{i}(t)\right) .
$$

Equations (3.2) are solved with a numerical scheme, e.g. Runge-Kutta, up to the next scattering time, or to $\Delta t$ if no scattering occurs.

\section{Scattering time}

The scattering process corresponding to equation (2.1) is Markovian. The distribution function of the random scattering time $\tau_{i}$ of particle $i$ is $(c f .(2.7))$

$$
\operatorname{Prob}\left(\tau_{i}<s\right)=1-\exp \left(-\int_{0}^{s} \lambda\left(k_{i}(t)\right) \mathrm{d} t\right) .
$$

According to the inverse transform method, the random scattering time is generated by solving the equation

$$
\int_{0}^{\tau_{i}} \lambda\left(k_{i}(t)\right) \mathrm{d} t=-\log u
$$

where $u \in[0,1]$ is a uniform random number. If (3.3) does not have a solution

$$
\tau_{i} \in[0, \Delta t],
$$

then the particle $i$ does not scatter during the time interval $[0, \Delta t]$. This happens with probability

$$
\operatorname{Prob}\left(\tau_{i} \geq \Delta t\right)=\exp \left(-\int_{0}^{\Delta t} \lambda\left(k_{i}(t)\right) \mathrm{d} t\right) .
$$

However, the integral on the left-hand side of (3.3) cannot be calculated analytically except in some trivial cases (e.g. if $\lambda$ is constant).

\section{Scattering event}

A scattering mechanism is chosen randomly according to the relative probabilities of all possible scattering mechanisms ( $c f .(2.8))$. A new state $k_{i}^{\prime}$ is generated from the differential cross section of this mechanism. After the scattering the electron can remain in the same valley (intravalley scattering) or be drawn into another valley (intervalley scattering).

\subsection{Generation of scattering times}

There are different ways of dealing with the problem of generating scattering times according to (3.3).

\subsubsection{Self scattering technique}

A common approach to the generation of scattering times is the introduction of fictitious scattering events. Whenever the self scattering is selected as the collision mechanism, nothing happens to the particle which maintains, after the scattering, the same energy and momentum it had before. The self scattering does not alter the statistical distribution of the real scattering events, but the (artificially increased) total scattering rate changes so that the integral in (3.3) can be handled. 


\section{Constant-Gamma scheme}

Let $\Gamma$ be a number greater then the largest scattering rate possible in the simulation, i.e.

$$
\Gamma \geq \max _{k \in \Omega} \lambda(k)
$$

When introducing the self scattering rate $\Gamma-\lambda(k)$, the total scattering rate becomes constant and the scattering time is obtained from (3.3) as

$$
\tau_{i}=-\frac{\log u}{\Gamma}
$$

\section{Piecewise-constant-Gamma scheme}

In order to reduce the number of self scattering events, particles are treated differently dependent on their initial energy. Consider, for example, numbers $\Gamma_{1}$ and $\Gamma_{2}$ such that

$$
\Gamma_{1} \geq \max _{\varepsilon(k) \leq \varepsilon_{1}} \lambda(k), \quad \Gamma_{2} \geq \max _{k \in \Omega} \lambda(k)
$$

where $\varepsilon_{1}$ is some energy level. Particles with energy above $\varepsilon_{1}$ are treated according to the constant-Gamma scheme with the upper bound $\Gamma_{2}$. Particles with energy below $\varepsilon_{1}$ are treated using the upper bound

$$
\Gamma(t)= \begin{cases}\Gamma_{1}, & \text { if } t \leq \tilde{t}_{i} \\ \Gamma_{2}, & \text { otherwise }\end{cases}
$$

where $\tilde{t}_{i}$ is the time when $\lambda\left(k_{i}(t)\right)$ first crosses the level $\Gamma_{1}$. When introducing the self scattering rate $\Gamma(t)-$ $\lambda\left(k_{i}(t)\right)$, the integral in equation (3.3) takes the form

$$
\int_{0}^{s} \Gamma(t) \mathrm{d} t= \begin{cases}s \Gamma_{1}, & \text { if } s \leq \tilde{t}_{i} \\ \tilde{t}_{i} \Gamma_{1}+\left(s-\tilde{t}_{i}\right) \Gamma_{2} & \text { otherwise }\end{cases}
$$

Thus, the scattering time is obtained as

$$
\tau_{i}= \begin{cases}-\frac{\log u}{\Gamma_{1}}, & \text { if }-\log u \leq \Gamma_{1} \tilde{t}_{i} \\ -\frac{\log u}{\Gamma_{2}}+\tilde{t}_{i}\left(1-\frac{\Gamma_{1}}{\Gamma_{2}}\right), & \text { otherwise }\end{cases}
$$

The constant-Gamma scheme is recovered for the choice $\Gamma_{1}=\Gamma_{2}$.

\section{Individual-Gamma scheme}

Here an individual upper bound is used for each particle. Consider a time increment

$$
t_{\text {inc }}=\frac{\Delta t}{L}, \quad \text { for some } \quad L=1,2, \ldots,
$$

and numbers $\Gamma_{i, l}$, for $i=1, \ldots, N$ and $l=1, \ldots, L$, such that

$$
\Gamma_{i, l} \geq \max _{t \in\left[t_{l-1}, t_{l}\right]} \lambda\left(k_{i}(t)\right), \quad \text { where } \quad t_{l}=l \Delta t_{\mathrm{sc}}
$$

Particles are treated using the upper bound

$$
\Gamma_{i}(t)=\Gamma_{i, l} \quad \text { if } \quad t \in\left[t_{l-1}, t_{l}\right], \quad l=1, \ldots, L,
$$


and the self scattering rate $\Gamma_{i}(t)-\lambda\left(k_{i}(t)\right)$. The integral in equation (3.3) takes the form

$$
\int_{0}^{s} \Gamma_{i}(t) \mathrm{d} t=\sum_{l=1}^{l(s)-1} \Gamma_{i, l} \Delta t_{\mathrm{inc}}+\Gamma_{i, l(s)}\left[s-(l(s)-1) \Delta t_{\mathrm{inc}}\right],
$$

where $l(s)$ is the index such that $s \in\left(t_{l(s)-1}, t_{l(s)}\right]$. Thus, the scattering time is obtained as follows:

- If there exists $l^{\prime}$ such that

$$
\Delta t_{\mathrm{inc}} \sum_{l=1}^{l^{\prime}-1} \Gamma_{i, l}<-\log u \leq \Delta t_{\mathrm{inc}} \sum_{l=1}^{l^{\prime}} \Gamma_{i, l}
$$

then

$$
\tau_{i}=\frac{1}{\Gamma_{i, l^{\prime}}}\left[-\log u-\Delta t_{\mathrm{inc}} \sum_{l=1}^{l^{\prime}-1} \Gamma_{i, l}\right]+\left(l^{\prime}-1\right) \Delta t_{\mathrm{inc}} .
$$

- If

$$
\Delta t_{\text {inc }} \sum_{l=1}^{L} \Gamma_{i, l}<-\log u,
$$

then there is no scattering during the time step $\Delta t$.

Note that the simple formula of the constant-Gamma scheme (cf. (3.6)) is recovered if $\Gamma_{i, l}=\Gamma_{i}$ for all $l=$ $1, \ldots, L$ (in particular, if $L=1$ ). However, the individual upper bound $\Gamma_{i}$ satisfies

$$
\Gamma_{i} \geq \max _{t \in[0, \Delta t]} \lambda\left(k_{i}(t)\right)
$$

instead of (3.5).

\subsubsection{Constant time technique}

This technique is based on a constant scattering time step $\Delta t_{\mathrm{sc}}$. Each particle is moved over the time step. Then it suffers a scattering with probability

$$
\min \left(1, \lambda\left(k_{i}\left(\Delta t_{\mathrm{sc}}\right)\right) \Delta t_{\mathrm{sc}}\right) .
$$

It is assumed that the scattering time step is small enough so that

$$
\lambda\left(k_{i}\left(\Delta t_{\mathrm{sc}}\right)\right) \Delta t_{\mathrm{sc}}<1, \quad i=1, \ldots, N .
$$

Probability (3.10) is obtained from (3.4) by the approximation

$$
\begin{aligned}
\exp \left(-\int_{0}^{\Delta t_{\mathrm{sc}}} \lambda\left(k_{i}(t)\right) \mathrm{d} t\right) & \simeq 1-\int_{0}^{\Delta t_{\mathrm{sc}}} \lambda\left(k_{i}(t)\right) \mathrm{d} t \\
& \simeq 1-\lambda\left(k_{i}\left(\Delta t_{\mathrm{sc}}\right)\right) \Delta t_{\mathrm{sc}}
\end{aligned}
$$

This approach is similar to the individual-Gamma scheme from Section 3.2.1, but here the scattering time is always a multiple of $\Delta t_{\mathrm{sc}}$. 


\subsection{Comments}

The idea of "self scattering" goes back to [12,13] (see also [3]). The notation $\Gamma$ was introduced for a constant upper bound of the total scattering rate. The term "Gamma" was used in [17] in order to give names to various modifications of the self scattering approach.

The piecewise-constant-Gamma scheme was introduced in [2] (see also [6], and [5], Sect. 3.4). The generalization to more than two pieces (named "variable $\Gamma$ scheme") was treated in [15]. The problem of optimizing the choice of pieces was addressed in [9]. Our description of the method is a slight generalization, where the standard case is obtained for $\Gamma_{1}=\tilde{\lambda}\left(\varepsilon_{1}\right)(c f .(3.7))$ provided that

$$
\lambda(k)=\tilde{\lambda}(\varepsilon(k))
$$

for an appropriate function $\tilde{\lambda}$.

The individual-Gamma scheme from Section 3.2.1 is basically identical to the "constant time method" studied in [17] as a special self scattering technique. A synchronized version (named "ensemble constant time technique") was described in [11]. In this paper we use a different name in order to have a clear distinction from the method of Section 3.2.2.

The constant time technique from Section 3.2.2 was introduced in [4] (see also [5], p. 251). It is implemented in the DAMOCLES code ( $c f$. [8], p. 19). The name was mentioned by Fischetti in a private communication.

\section{Setup of THE NUMERICAL EXPERIMENTS}

\subsection{Test case}

We consider a $n^{+}-n-n^{+}$silicon diode, which is the simplest one-dimensional inhomogeneous structure. This device has often been simulated when new device modeling techniques or transport theories are discussed, because its internal potential bears resemblance to the potential in a MOSFET channel or bipolar junction transistor active region. The diode consists of two highly doped regions $n^{+}$(called cathode and anode) connected by a less doped region $n$ called channel. In our simulations, the $n^{+}$regions are $0.15 \mu \mathrm{m}-$ long doped to a density of $2 \times 10^{17} \mathrm{~cm}^{-3}$, while the channel $n$ is $0.25 \mu \mathrm{m}$-long doped with a density of $10^{15} \mathrm{~cm}^{-3}$. We consider the device at room temperature $T_{0}=300 \mathrm{~K}$ and $1 \mathrm{~V}$ of applied bias. In order to avoid boundary effects, we adopt periodic boundary conditions so that an electron that exits through an interface is reinjected with the same velocity at the other end of the device.

Several functionals of the solution are calculated, namely the mean velocity, the mean energy, the mean density, the electric field, the potential, and the density current (averaged over all spatial cells). To skip

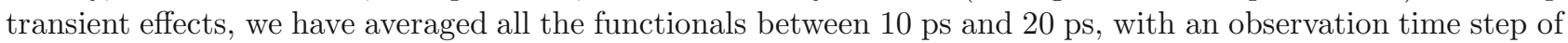
0.1 ps. In order to construct confidence intervals, $N_{r}$ independent runs are performed for each set of parameters.

The results were obtained in the GRID environment COMETA, where the CPU of each node is an opteron bi-processor dual core.

\subsection{Error measurements}

Consider some functional (velocity, energy, ...)

$$
F_{i}\left(x_{j}, N, \Delta t\right), \quad i=1, \ldots, N_{r}
$$

where $x_{j}, j=1, \ldots, C$, indicates the spatial cell and $N, \Delta \mathrm{t}$ are the parameters (particle number, time step) with respect to which convergence is studied. A reference solution $F^{\text {ref }}\left(x_{j}\right)$ represents the best available dataset. 
In order to study the rate of convergence, we introduce the following measures of error depending on the type of the considered functional:

- Average absolute error (AAE)

For each cell $x_{j}$, the absolute error between the functional (4.1) and the reference solution $F^{\text {ref }}\left(x_{j}\right)$ is measured. Then the average is taken over the cells, i.e.

$$
\operatorname{AAE}_{i}(N, \Delta t)=\frac{1}{C} \sum_{j=1}^{C}\left|F_{i}\left(x_{j}, N, \Delta t\right)-F^{\mathrm{ref}}\left(x_{j}\right)\right| .
$$

- Fractional truncation error (FTE)

For functionals that do not depend on the cell (e.g. current density), we introduce

$$
\operatorname{FTE}_{i}(N, \Delta t)=\frac{\left|F_{i}(N, \Delta t)-F^{\mathrm{ref}}\right|}{\left|F^{\mathrm{ref}}\right|} .
$$

\subsection{Confidence intervals}

Let $\xi_{i}, i=1, \ldots, N_{r}$, be a quantity obtained with independent runs (repetitions). The confidence interval is evaluated as

$$
\frac{1}{N_{r}} \sum_{i=1}^{N_{r}} \xi_{i} \pm 3 \sqrt{\frac{1}{N_{r}}\left(\frac{1}{N_{r}} \sum_{i=1}^{N_{r}} \xi_{i}^{2}-\left[\frac{1}{N_{r}} \sum_{i=1}^{N_{r}} \xi_{i}\right]^{2}\right)}=\mu \pm \sigma
$$

where the factor 3 corresponds to a $99.7 \%$ confidence level.

Confidence intervals for the average absolute error are obtained via (4.4) with (cf. (4.2))

$$
\xi_{i}(N, \Delta \mathrm{t})=\operatorname{AAE}_{i}(N, \Delta \mathrm{t}) .
$$

Confidence intervals for the fractional truncation error are obtained via (4.4) with (cf. (4.3))

$$
\xi_{i}(N, \Delta \mathrm{t})=\operatorname{FTE}_{i}(N, \Delta \mathrm{t}) .
$$

\section{Results}

In the first part we study the systematic error introduced by the finite number of particles $N$ and by the splitting time step $\Delta t$. We consider the constant-Gamma self scattering technique (SST) from Section 3.2.1 and the constant time technique (CTT) from Section 3.2.2. A second-first order Runge-Kutta numerical integrator is used in the CTT ( $c f$. [8], p. 11). In this part the parameter choice is $\Delta t_{\mathrm{sc}}=\Delta t$. Both a second-first order Runge-Kutta and a second order Runge-Kutta method are used in the SST.

In the second part we study issues related to the efficiency. As to the SST, the number of self-scatterings is considered. The constant-Gamma scheme is compared with the piecewise-constant-Gamma scheme and the individual-Gamma scheme. The systematic error related to the reduction of self scattering events is pointed out. As to the CTT, the scattering time step $\Delta t_{\mathrm{sc}}$ is taken smaller than the field-adjusting time step $\Delta t$.

\subsection{Studies of the systematic error}

The error of the Monte Carlo algorithms consists of two components, the systematic error (wrong expectation) and the statistical error (fluctuating estimate of expectation). Two numerical parameters are involved, the particle number $N$ and the time step $\Delta t$. The particle number influences both the systematic and statistical errors. 
The reference solution $F^{\text {ref }}$, which will be called master curve, is obtained using SST with $N=620000$ particles, time step $\Delta t=0.001 \mathrm{ps}$ and $N_{r}=10$ repetitions.

\subsubsection{Particle number error}

The origin of this systematic error is in the calculation of the internal electric field. At this stage the particles interact with each other, otherwise they are independent. The dependence vanishes, when the number of particles goes to infinity.

We calculate several functionals with both the CTT and the SST for different values of the particle number $N$. In order to ensure the same level of fluctuations, the number of repetitions $N_{r}$ is chosen such that $N_{r} \times N=6400$. The time step is $\Delta t=0.001 \mathrm{ps}$.

In Figure 1 we plot the functionals "mean velocity" and "mean energy" obtained with the CTT.

The corresponding average absolute error is plotted in Figure 2 for CTT and SST. The behavior of the error is the same for both methods. The deviation at the end is due to the fact that CTT involves a time step error even for $\Delta \mathrm{t}=0.001 \mathrm{ps}$. The confidence intervals are not displayed because they are less than the size of the symbols.

\subsubsection{Time step error}

This systematic error has different sources:

A: Error due to the wrong electric field, which is updated only at the end of the time step (splitting error);

B: Error due to the wrong particle trajectory, which is obtained by an ODE solver;

$\mathrm{C}$ : Error due to the wrong scattering time evolution in the CTT.

We calculate several functionals with both the CTT and the SST for different values of the time step $\Delta t$. The particle number is $N=62000$.

In Figures 3 and 4 we plot the results obtained with SST and CTT, respectively, for the velocity and energy. The AAE corresponding to these fields is plotted in Figure 5. The confidence intervals are smaller than the size of the symbols. The results for other functionals show similar behavior.

For some functionals the confidence interval for CTT does not cover the master value even for the smallest time step used so far. Results for the average density current obtained using CTT with further decreased time steps are given in Figure 6, where the FTE corresponding to this scalar quantity is plotted.

The figures clearly illustrate the different error sources mentioned above. The error of type C is present only in the CTT. The significance of this contribution can be seen by comparing the curves for CTT and SST with second-first order Runge-Kutta in Figure 5. In Figure 7, we plot the average number of real scattering events per particle per time, versus the time step $\Delta t$, obtained using CTT and SST. Even when the number of scattering events is correct $(\Delta t \leq 0.02 \mathrm{ps})$, there is still a considerable error due to the wrong moments of scattering in the CTT.

The error of type B can be detected by comparing the SST with second-first order Runge-Kutta and second order Runge-Kutta in Figure 5.

Finally, it can be seen that the error of type A has vanished when the time step is less than 0.05 ps. This is consistent with the following recommendation.

Remark 5.1. An electron gas can develop plasma oscillations with angular frequency given by (cf. [4], p. 9736, or [5], p. 252)

$$
\omega_{p}=\sqrt{\frac{q^{2} n}{\epsilon m^{\star}}} .
$$

In order to avoid instabilities related to these oscillations, the time step must be chosen considerably smaller than the inverse of $\omega_{p}$, i.e.

$$
\Delta \mathrm{t}<\frac{1}{\omega_{p}} \simeq 0.076 \mathrm{ps},
$$

where the number has been evaluated for the high doping region of the device described in Section 4.1. 

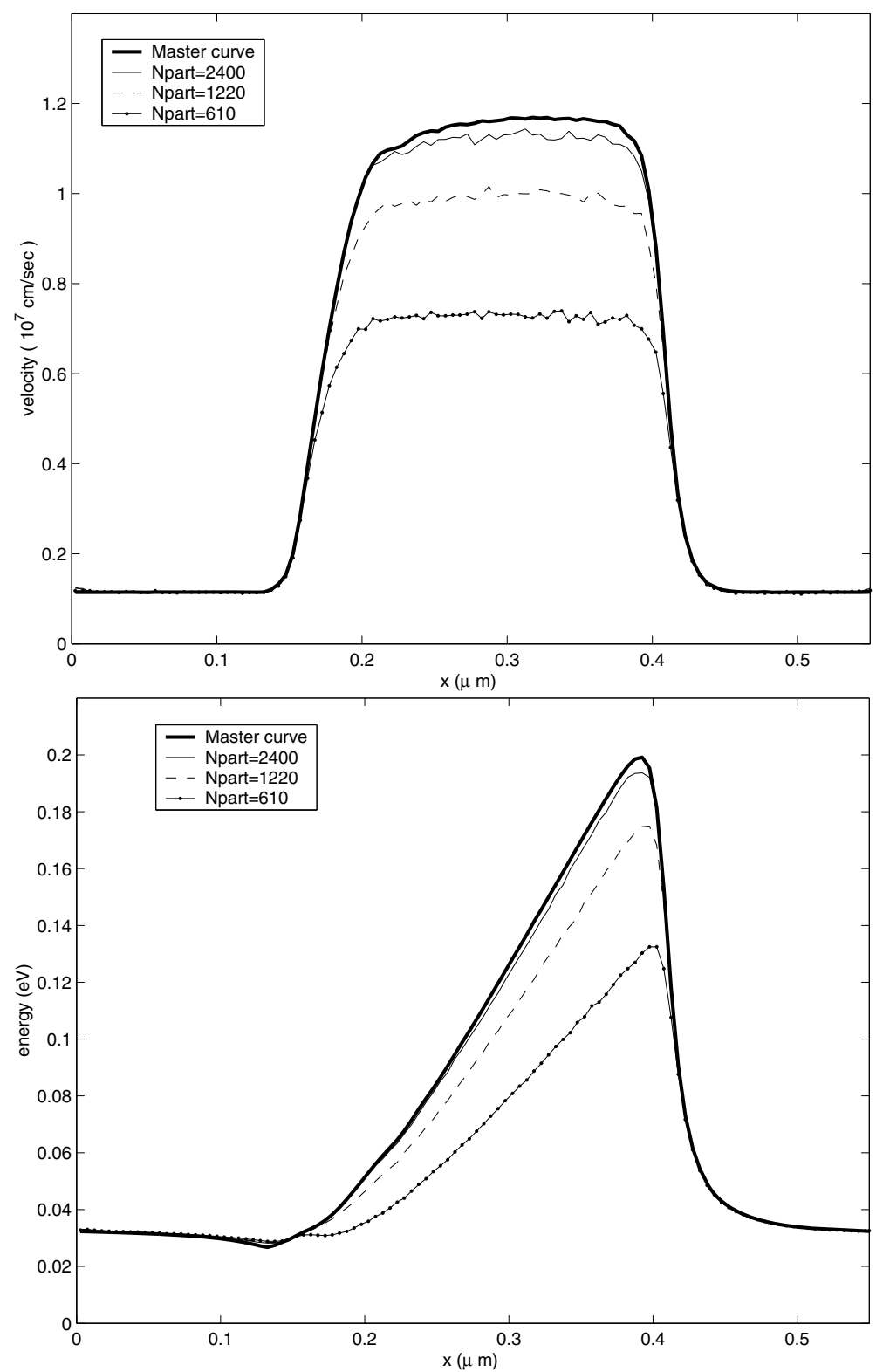

FIGURE 1. Mean velocity (top) and mean energy (bottom) as functions of the position, obtained with the CTT for different particle numbers.

\subsection{Issues related to the efficiency}

Here we address the problem of optimization. As to the CTT, several choices of $\Delta t_{\mathrm{sc}}<\Delta t$ are checked. As to the SST, various methods of reducing the number of self scattering events are considered. Finally, we compare the CPU times for different algorithms. 

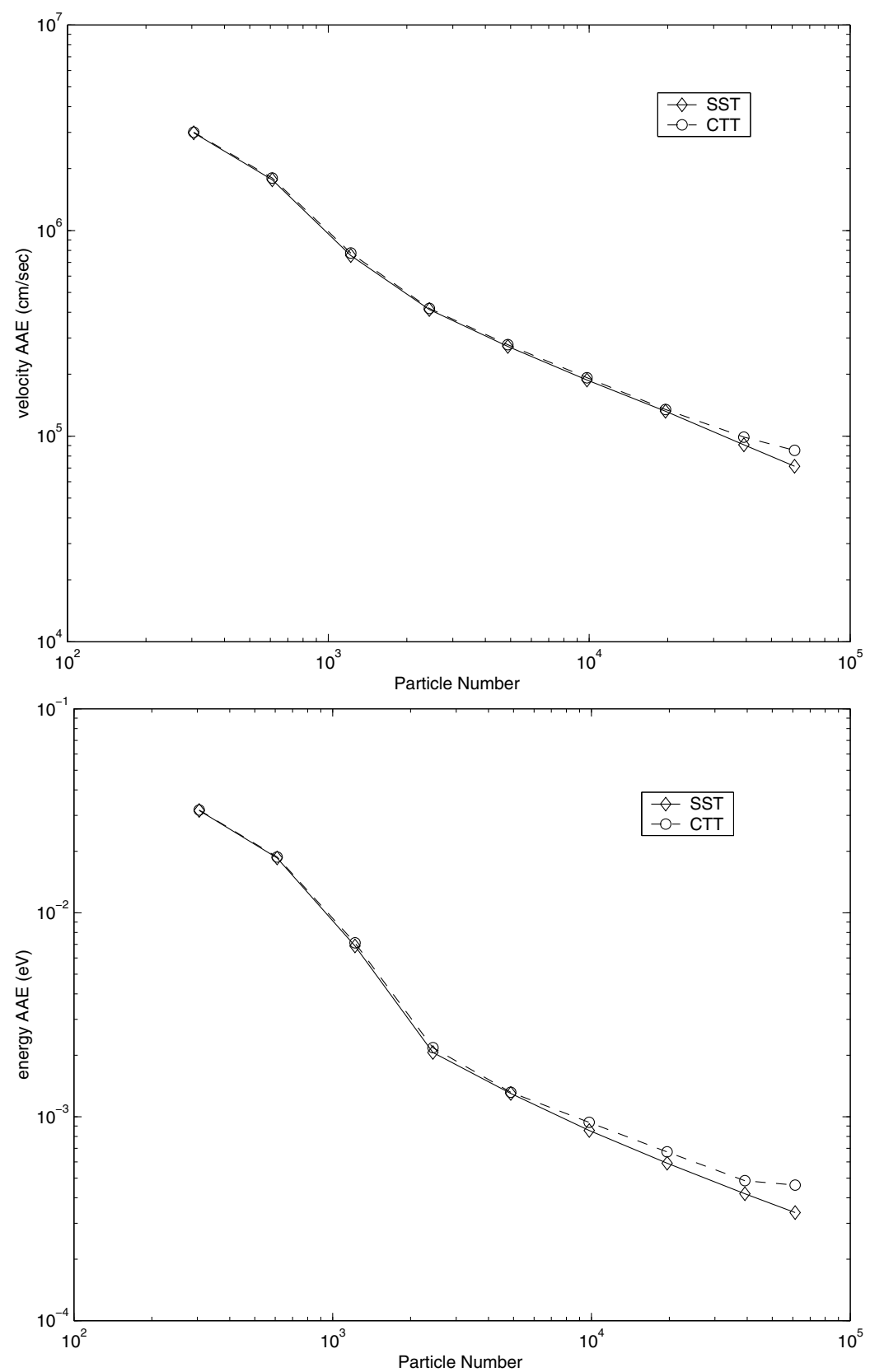

Figure 2. Average absolute error for the mean velocity (top) and the mean energy (bottom) versus the particle number, obtained with the SST and with the CTT.

\subsubsection{Self scattering technique}

In the constant-Gamma scheme the value

$$
\Gamma=119.2 \mathrm{ps}^{-1}
$$



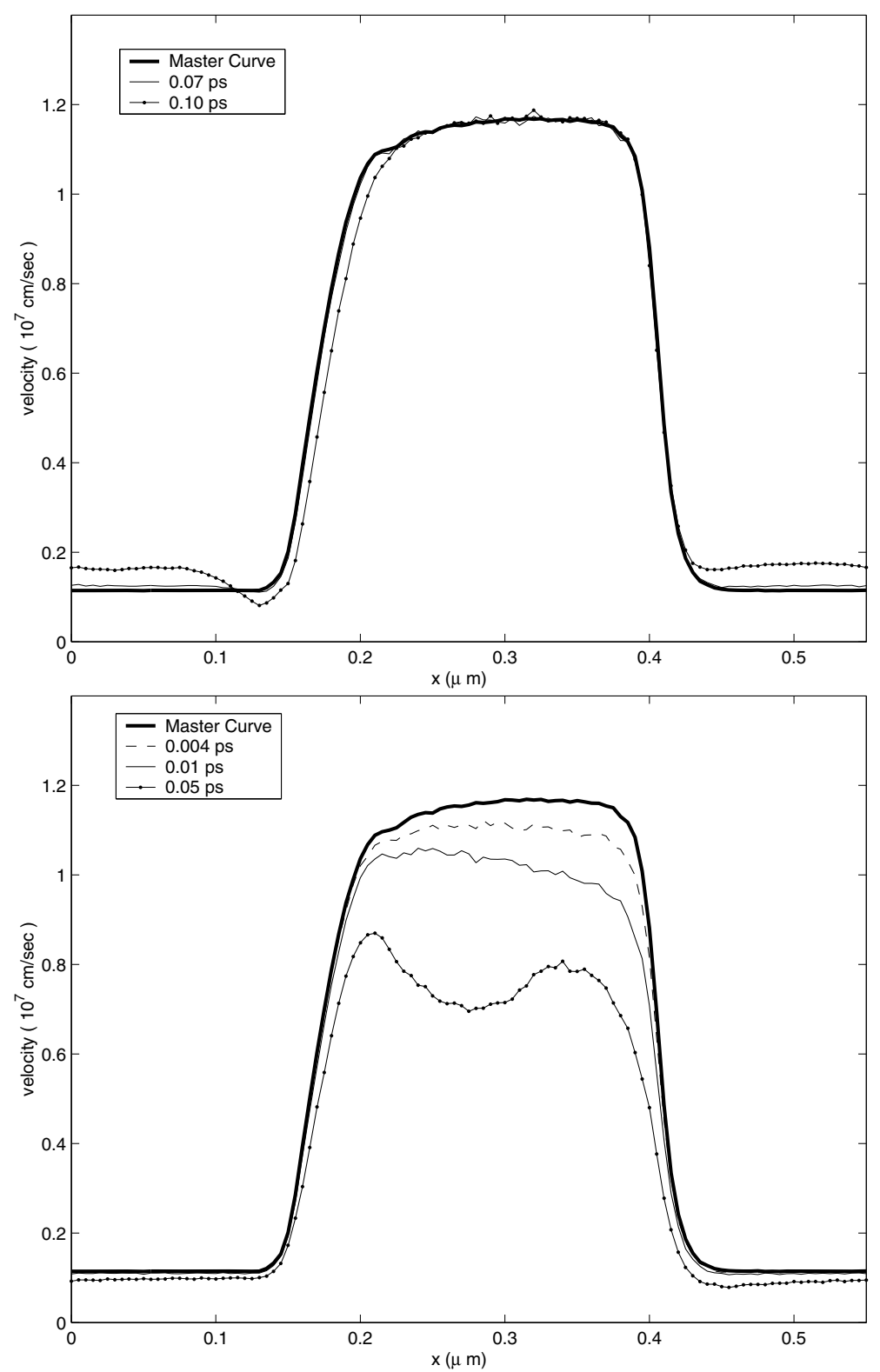

Figure 3. Mean velocity as a function of the position, obtained with the SST (top) and the CTT (bottom) for different time steps.

is used, which corresponds to an energy of $1 \mathrm{eV}$. The average time step between scattering events is $\Gamma^{-1}=$ $0.0084 \mathrm{ps}$.

Figure 8 shows the energy distribution among particles. Accordingly, we choose $\varepsilon_{1}=0.1 \mathrm{eV}(c f .(3.7))$ in the piecewise-constant-Gamma scheme so that about $95 \%$ of the particles have an energy below that level. We consider $\Gamma_{1}=\tilde{\lambda}\left(\varepsilon_{2}\right)(c f .(3.11))$, for $\varepsilon_{2}=0.1,0.2,0.4,1.0 \mathrm{eV}$. The choice $\varepsilon_{2}=1.0 \mathrm{eV}$ gives the constant-Gamma scheme, while the choice $\varepsilon_{2}=0.1 \mathrm{eV}$ provides the standard version of the piecewise-constant-Gamma scheme.

In the individual-Gamma scheme we choose $L=1$ in (3.9). The individual upper bound $\Gamma_{i}$ is determined by making the Runge-Kutta step over $\Delta t$ and taking the maximum energy. 

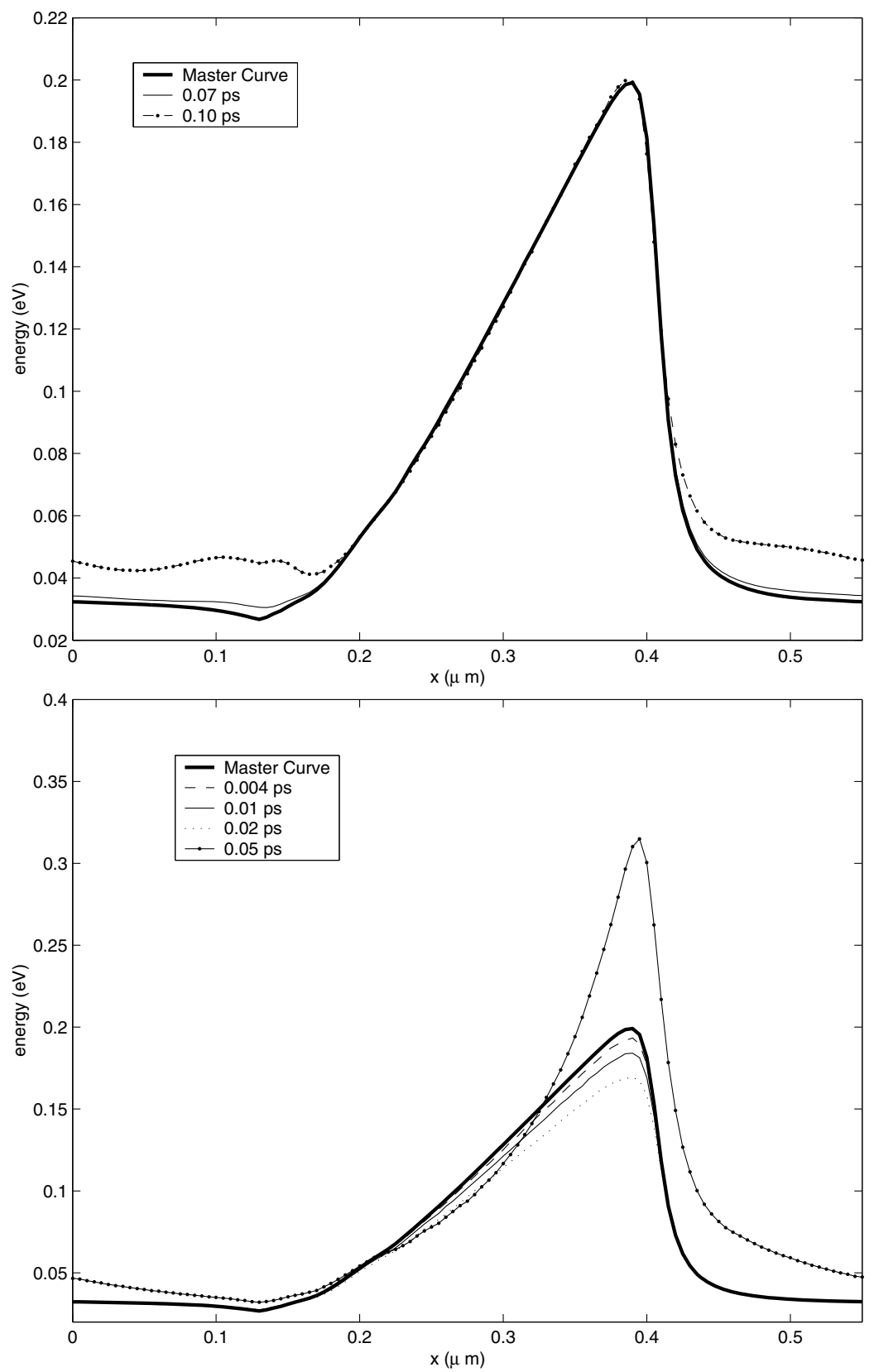

FIgURE 4. Mean energy as a function of the position, obtained with the SST (top) and the CTT (bottom) for different time steps.

Table 1 provides the average scattering numbers per particle per time. Note that the data in the first line of Table 1 are consistent with the quantity (5.1), which represents the average number of scattering events (including self scattering).

Next we study the additional systematic error introduced by the schemes that reduce the number of self scatterings. 

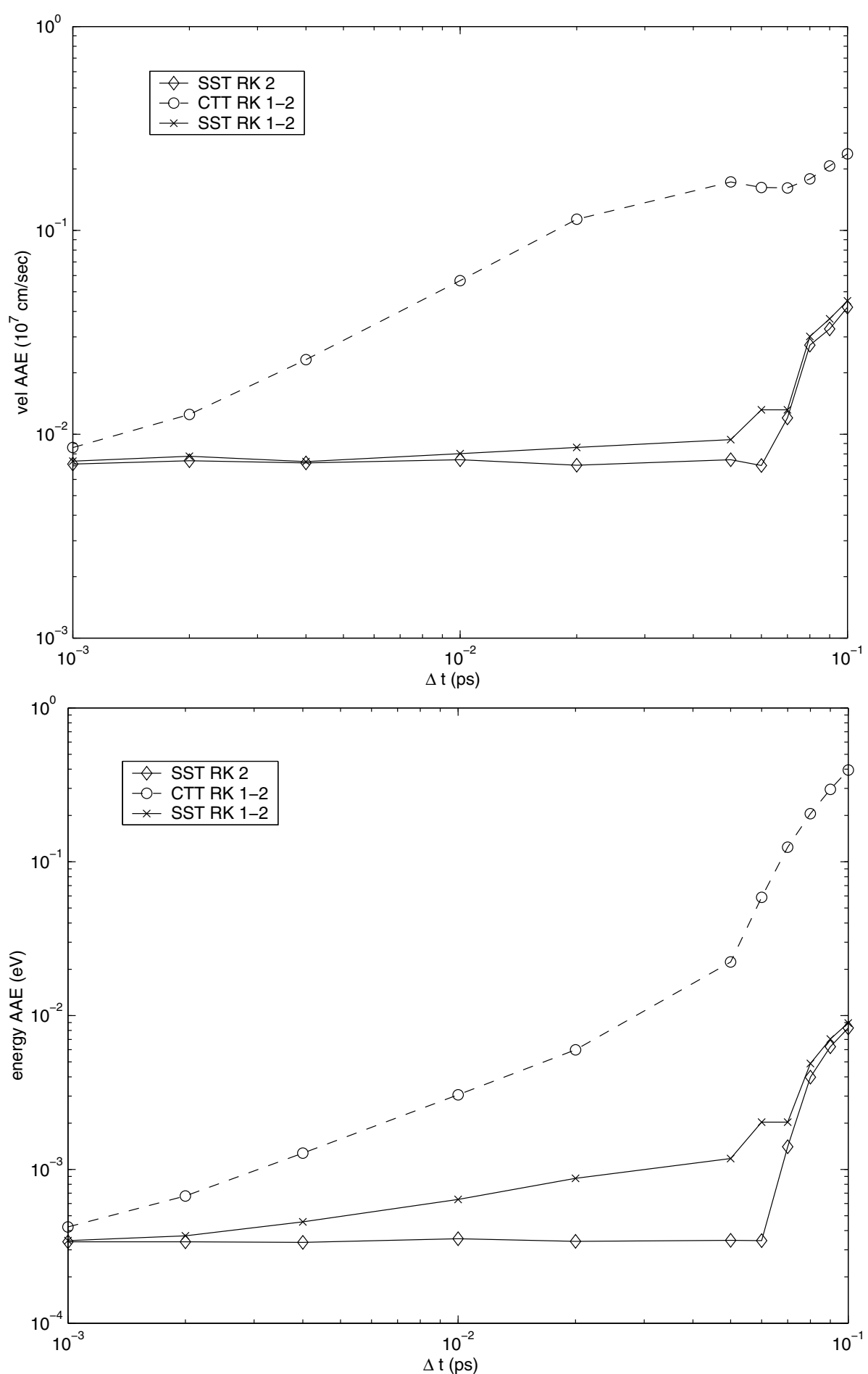

Figure 5. Average absolute error for the mean velocity (top) and the mean energy (bottom) as a function of the time step, obtained with the CTT and with the SST (star symbol: Runge Kutta order 1-2, diamond symbol: Runge Kutta order 2). 


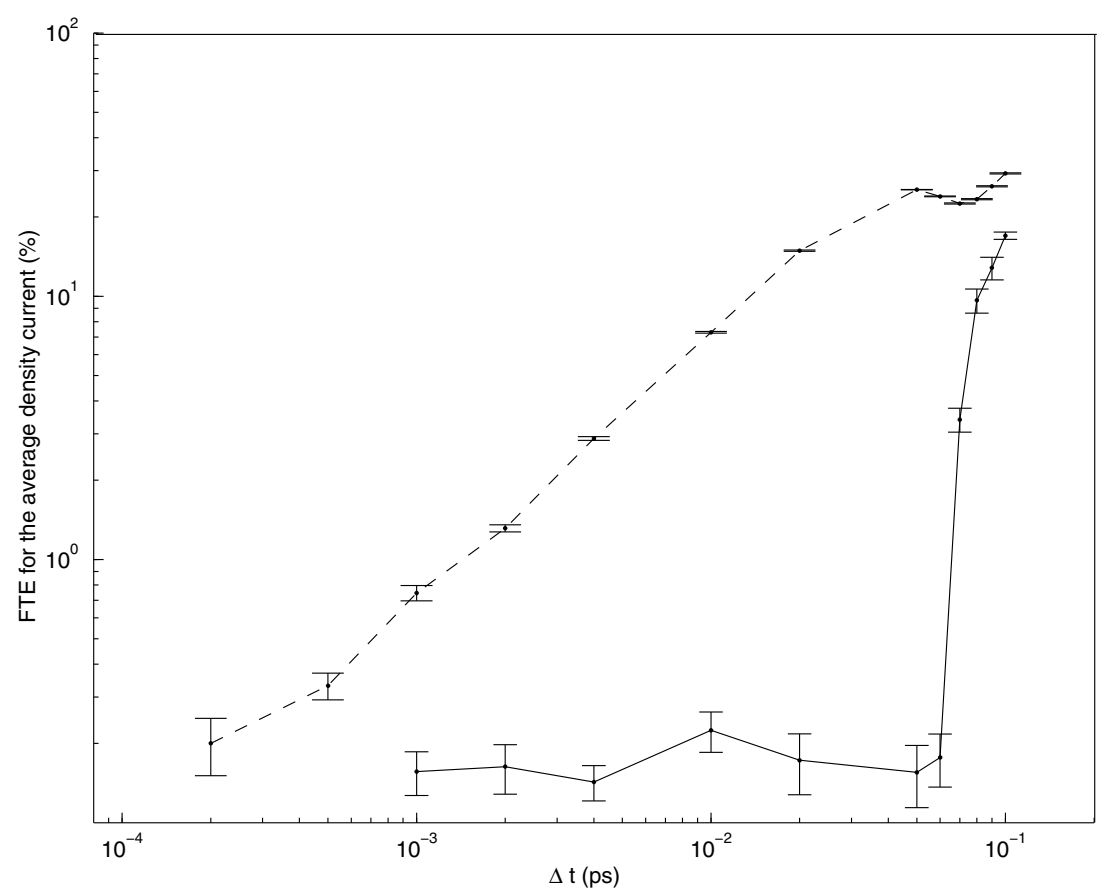

FiguRE 6. Fractional truncation error (with confidence intervals) for the average density current versus the time step, obtained with the SST and the CTT.

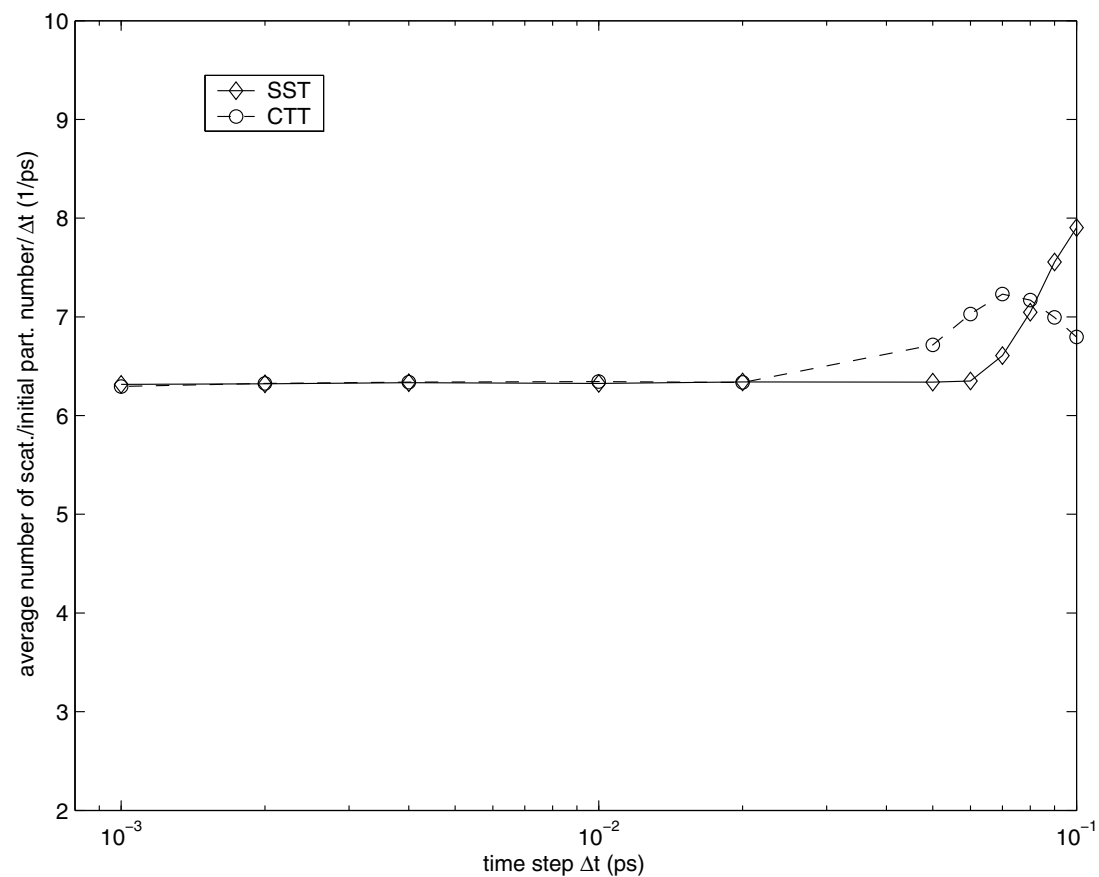

FiguRE 7. Average number of real scattering events per particle per time versus the time step, obtained with the SST and the CTT. 


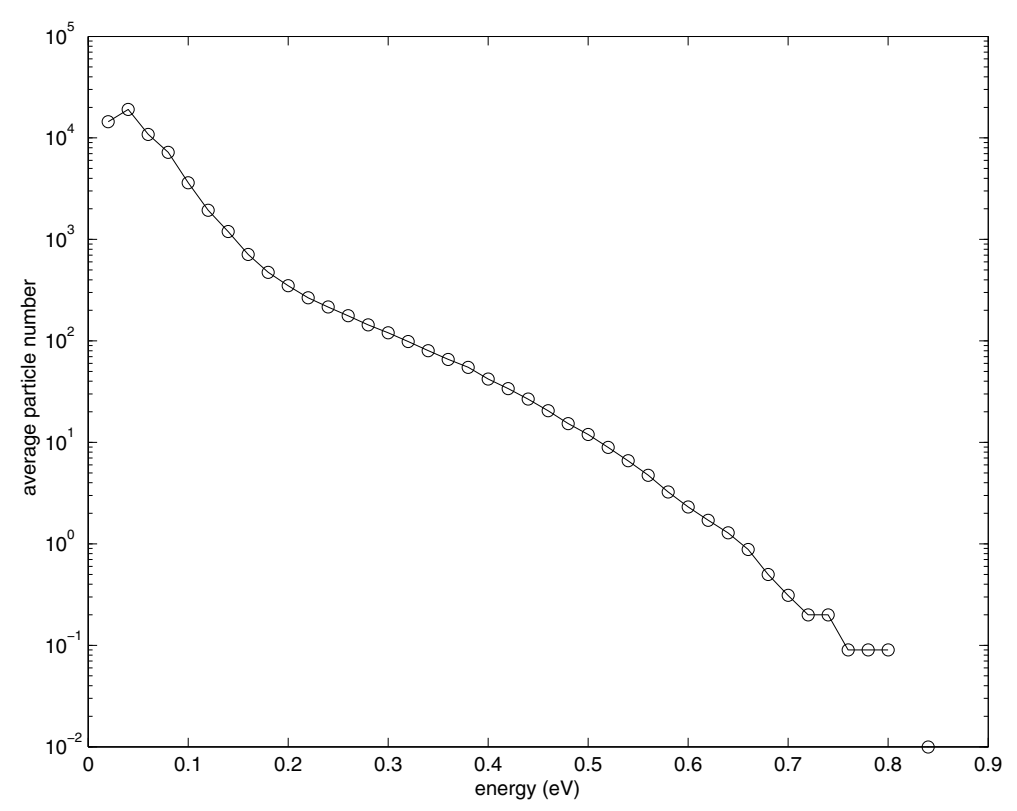

FiguRE 8. Average particle number versus energy.

TABLE 1. Average number of scattering events per particle per time (in ps).

\begin{tabular}{|c|c|c|c|}
\hline Algorithm & RealScat & SelfScat & SS percentage \\
\hline Constant-Gamma & 6.3 & 113 & $95 \%$ \\
\hline p-c-Gamma $\Gamma_{1}(0.4)$ & 6.3 & 45.1 & $88 \%$ \\
\hline p-c-Gamma $\Gamma_{1}(0.2)$ & 6.3 & 26.5 & $81 \%$ \\
\hline p-c-Gamma $\Gamma_{1}(0.1)$ & 6.3 & 16.4 & $72 \%$ \\
\hline Individual-Gamma & 6.3 & 0.12 & $2 \%$ \\
\hline
\end{tabular}

\section{Piecewise-constant-Gamma scheme}

The times $\tilde{t}_{i}(c f .(3.8))$ are calculated by a linear interpolation. Alternatively, one can determine $\tilde{t}_{i}$ by solving the equations of motion more precisely, but this would consume further CPU time.

Figure 9 shows the systematic error introduced by this procedure, when calculating the average energy (with $\Delta t=0.02 \mathrm{ps}$ ). The error disappears, when the energy level $\varepsilon_{2}$ is increased. We have also counted the corresponding numbers of $\tilde{t}_{i}$-calculations. They show how often a particle starting with an energy below $\varepsilon_{1}$ has an energy above $\varepsilon_{2}$ at the end of the time step. These numbers decrease with an increasing energy level and are almost zero for $\varepsilon_{2}=0.4 \mathrm{eV}$.

\section{Individual-Gamma scheme}

Figure 10 shows the percentage of scattering times outside the time step. In all these cases the Runge-Kutta prediction is used for making the free flow step.

We have also counted the number of cases where the energy calculated inside the time step is indeed bigger than the individual upper bound. This number grows with the time step so that some systematic error may occur. However, in the present test case it remains sufficiently small, and the individual-Gamma scheme does not produce any visible systematic error for the time steps $0.02 \mathrm{ps}$ and $0.05 \mathrm{ps}$. 


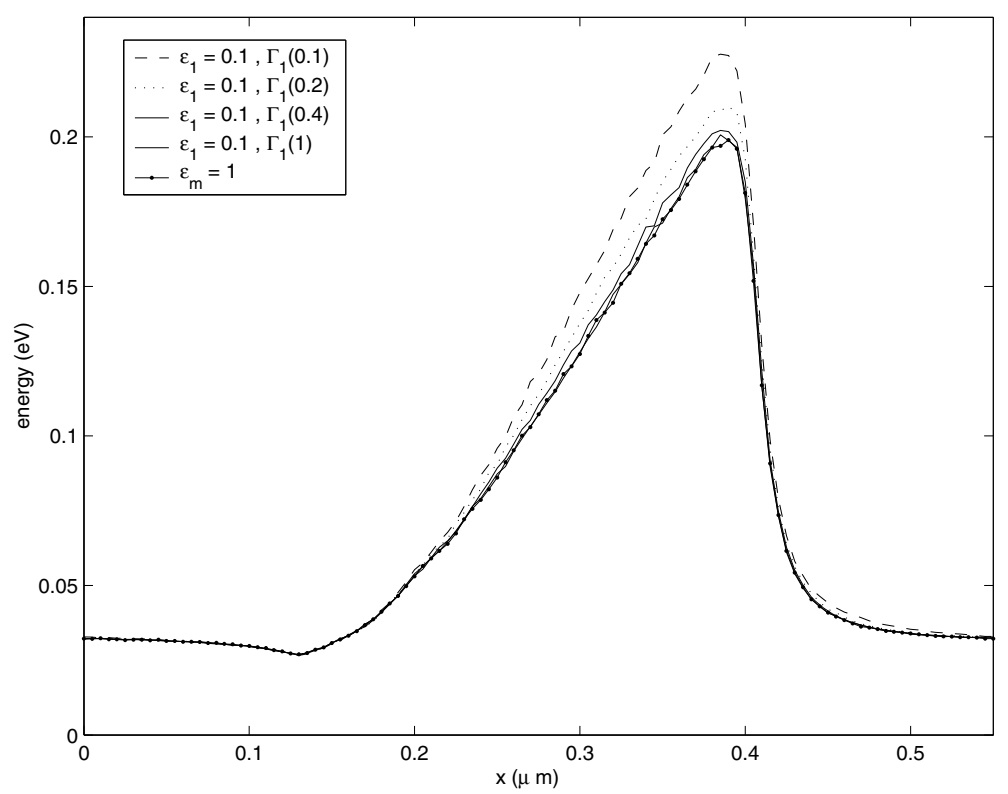

Figure 9. Mean energy as a function of the position, obtained with the piecewise-constantGamma scheme for different values of the energy level $\varepsilon_{2}$.

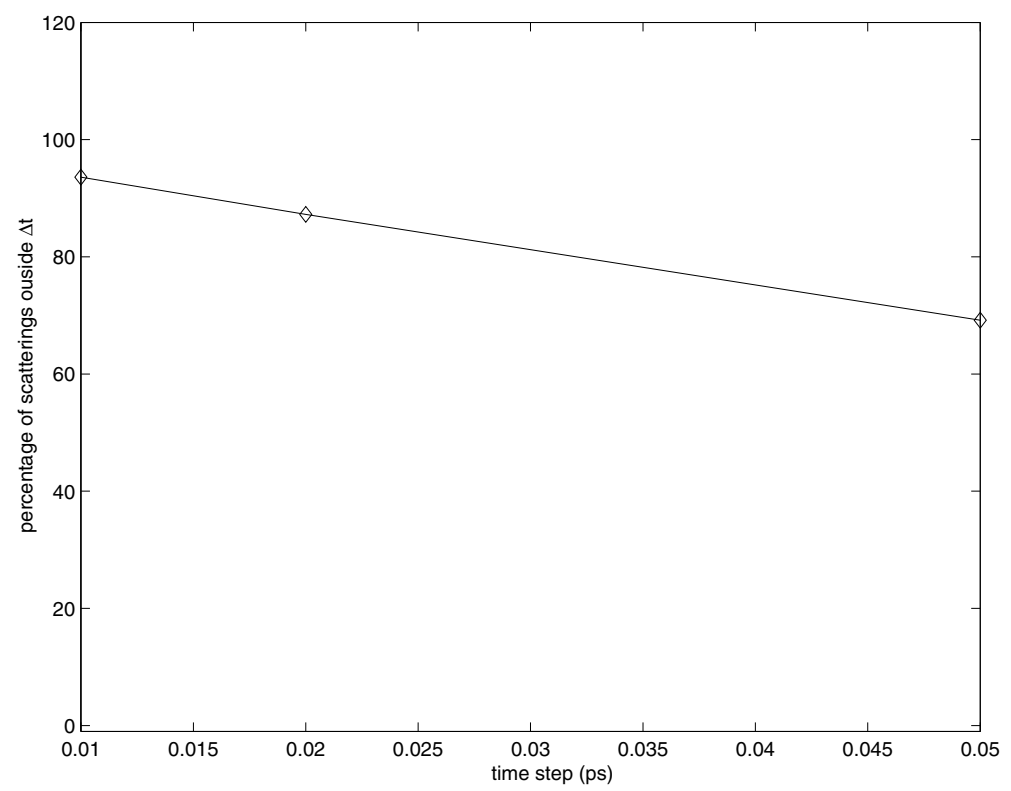

Figure 10. Percentage of scatterings outside the time step, obtained for the individual-Gamma scheme with different time steps.

\subsubsection{Constant time technique}

We have fixed $\Delta t_{\mathrm{sc}}=0.001 \mathrm{ps}$ and compared the cases $\Delta t=0.001 \mathrm{ps}$ with $\Delta t=0.02 \mathrm{ps}$ and $\Delta t=0.05$ ps. Results for the mean velocity are displayed in Figure 11. Other functionals show similar behavior. There is no visible additional error for $\Delta t=0.02 \mathrm{ps}$, but considerable error occurs for $\Delta t=0.05 \mathrm{ps}$. 


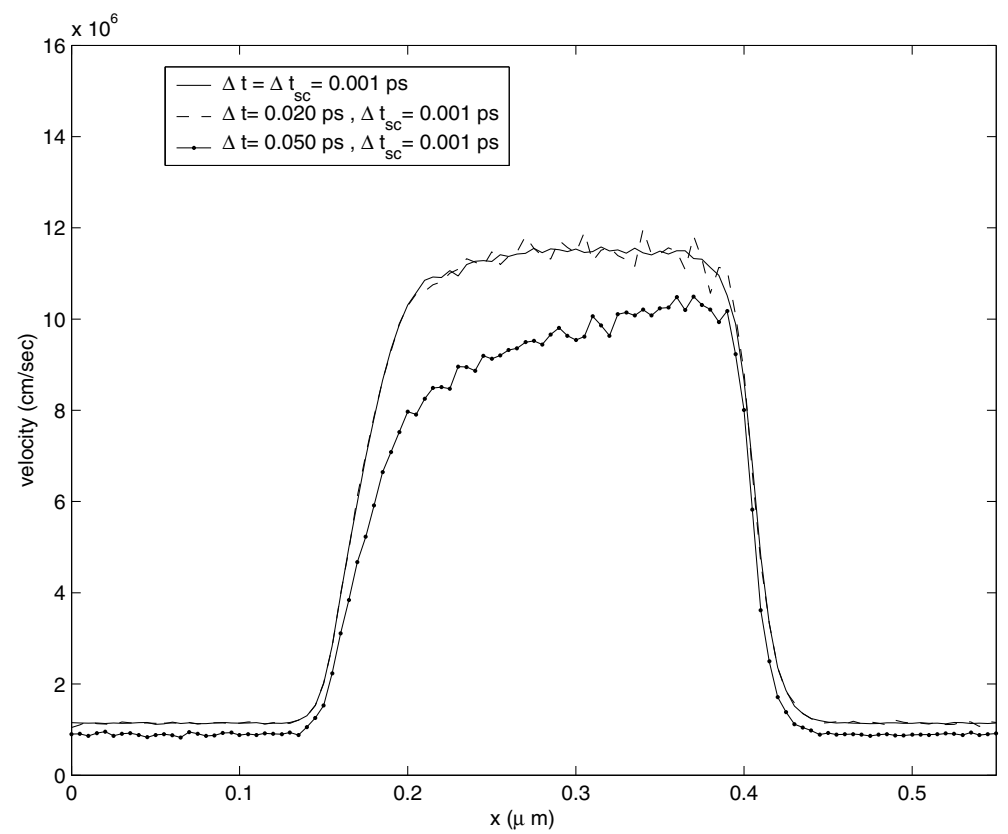

FIGURE 11. Mean velocity as a function of the position, obtained with the CTT for different field-adjusting time steps.

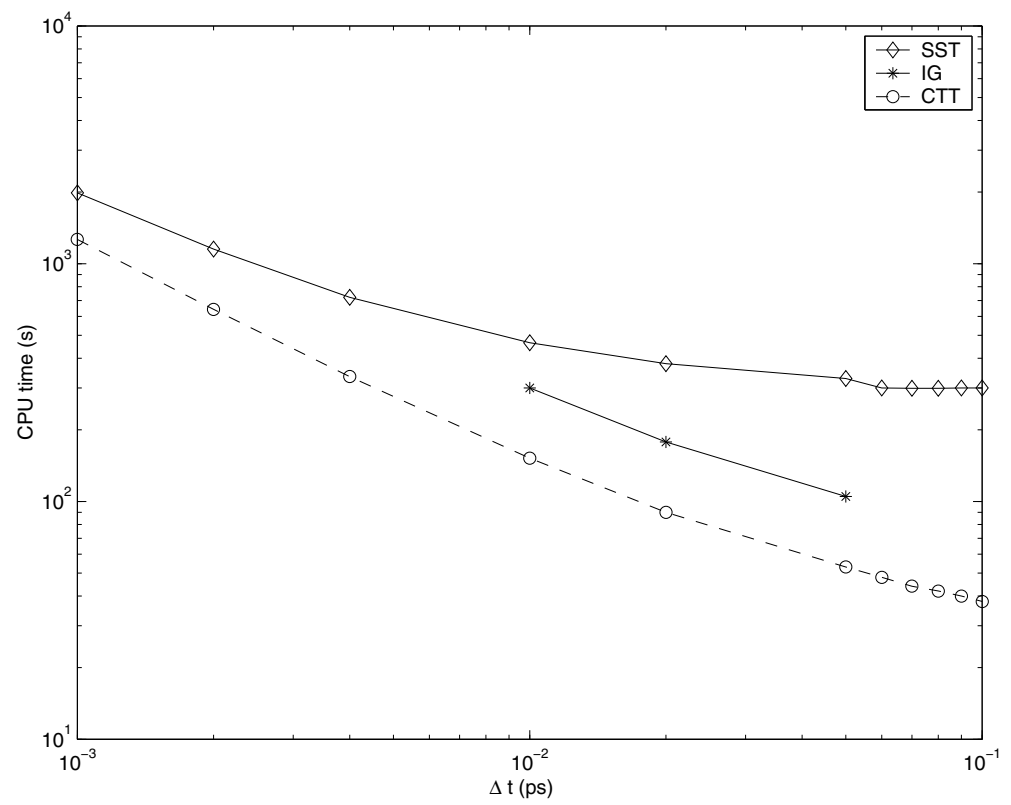

Figure 12. CPU times versus the time step, obtained for different methods with particle number $N=62000$ and one repetition. 


\subsubsection{Comparison}

Figure 12 provides the CPU times for the methods (SST and CTT) used for the study of the systematic error in Section 5.1, and for the individual-Gamma scheme (IG).

The efficiency gain factor represents the relation between the CPU times for two methods needed to obtain a given accuracy. According to Figure 12, there is an efficiency gain of at least one order of magnitude, when comparing the individual-Gamma scheme (with $\Delta t=0.05 \mathrm{ps}$ ) and the CTT (with $\Delta t=0.001 \mathrm{ps}$ ), which produce a similar systematic error ( $c f$. Fig. 5).

The efficiency gain factor depends on the class of calculated functionals and on the desired precision of the results. For example, the above mentioned efficiency gain between the individual-Gamma scheme and the CTT would further increase, when considering the functional studied in Figure 6. According to Figure 12, the gain factor, when comparing the individual-Gamma scheme and the constant-Gamma scheme, grows from about 1.5 for $\Delta t=0.01 \mathrm{ps}$ to more than 3 for $\Delta t=0.05 \mathrm{ps}$ (both methods produce the same systematic error).

Other observations not displayed in the figure were as follows. There is a gain of about $30 \%$ in CPU time, when comparing the piecewise-constant-Gamma scheme with $\Delta t=0.02 \mathrm{ps}$ and $\varepsilon_{2}=0.4 \mathrm{eV}$ (when the additional systematic error is negligible, cf. Fig. 9) and the constant-Gamma scheme. There is no significant reduction in the CPU time for the CTT (from $1278 \mathrm{~s}$ to $1226 \mathrm{~s}$ ), when comparing the cases $\Delta t=\Delta t_{\mathrm{sc}}=0.001 \mathrm{ps}$ and $\Delta t=0.02 \mathrm{ps}, \Delta t_{\mathrm{sc}}=0.001 \mathrm{ps}$ (when the additional systematic error is negligible, $c f$. Fig. 11).

\section{Conclusions}

The convergence behavior of various Monte Carlo schemes for semiconductors has been studied in the case of a $n^{+}-n-n^{+}$silicon diode. A detailed analysis of the systematic error with respect to numerical parameters was performed.

Errors due to the calculation of the internal electric field vanish rather rapidly. This applies both to the error related to the finite particle number and to the splitting time step error. The error due to the approximation of the particle trajectories is negligible, when a high order ODE solver is used.

The wrong scattering times are the most significant source of error. This type of error is present only in the constant time technique. It is reduced considerably when the scattering time step is taken smaller than the field-adjusting time step. This procedure simply brings the method closer to the self scattering technique. An analogous situation is observed in the case of the classical Boltzmann equation from rarefied gas dynamics for the Nanbu-scheme in relation to the Bird-scheme (see [14], Sects. 3.5.4 and 3.5.5).

A performance comparison of the algorithms has been carried out. The systematic error related to various efficiency improving schemes was analyzed. For the particular one-dimensional device considered, the self scattering technique (with a second order Runge-Kutta solver and individual majorants) turns out to be more efficient than the constant time technique (by one order of magnitude). Clearly the quantitative statements about efficiency depend on the test case. Moreover, CPU time measurements are computer and implementation dependent. However, the results concerning the systematic error and the number of self-scattering events provide useful indicators for further efficiency studies.

Acknowledgements. The first author has been supported by MIUR PRIN 2006 "Modelli per il trasporto di cariche nei semiconduttori: aspetti analitici e computazionali", "Progetti di ricerca di Ateneo", Università degli Studi di Catania, PI2S2 Project managed by the COMETA Consortium, the EU Marie Curie RTN project COMSON grant No. MRNTCT-2005-019417.

\section{REFERENCES}

[1] A.M. Anile and O. Muscato, Improved hydrodynamical model for carrier transport in semiconductors. Phys. Rev. B 51 (1995) $16728-16740$.

[2] V. Borsari and C. Jacoboni, Monte Carlo calculations on electron transport in CdTe. Phys. Stat. Sol. (B) 54 (1972) 649-662. 
[3] W. Fawcett, A.D. Boardman and S. Swain, Monte Carlo determination of electron transport properties in gallium arsenide. J. Phys. Chem. Solids 31 (1970) 1963-1990.

[4] M.V. Fischetti and S.E. Laux, Monte Carlo analysis of electron transport in small semiconductor devices including bandstructure and space-charge effects. Phys. Rev. B 38 (1988) 9721-9745.

[5] C. Jacoboni and P. Lugli, The Monte Carlo Method for Semiconductor Device Simulation. Springer, New York (1989).

[6] C. Jacoboni and L. Reggiani, The Monte Carlo method for the solution of charge transport in semiconductors with applications to covalent materials. Rev. Modern Phys. 55 (1983) 645-705.

[7] C. Jungemann and B. Meinerzhagen, Hierarchical Device Simulation. The Monte-Carlo Perspective. Springer, Wien (2003).

[8] S.E. Laux, M.V. Fischetti, Numerical aspects and implementation of the DAMOCLES Monte Carlo device simulation program, in Monte Carlo Device Simulation: Full Band and Beyond, K. Hess Ed., Kluwer, Boston (1991) 1-26.

[9] J.M. Miranda, C. Lin, M. Shaalan, H.L. Hartnagel and J.L. Sebastian, Influence of the minimization of self-scattering events on the Monte Carlo simulation of carrier transport in III-V semiconductors. Semicond. Sci. Technol. 14 (1999) 804-808.

[10] O. Muscato and W. Wagner, Time step truncation in direct simulation Monte Carlo for semiconductors. Compel 24 (2005) $1351-1366$

[11] U. Ravaioli, Vectorization of Monte Carlo algorithms for semiconductor simulation, in Monte Carlo Device Simulation: Full Band and Beyond, K. Hess Ed., Kluwer, Boston (1991) 267-284.

[12] H.D. Rees, Calculation of steady state distribution functions by exploiting stability. Phys. Lett. A 26 (1968) 416-417.

[13] H.D. Rees, Calculation of distribution functions by exploiting the stability of the steady state. J. Phys. Chem. Solids 30 (1969) 643-655.

[14] S. Rjasanow and W. Wagner, Stochastic Numerics for the Boltzmann Equation. Springer, Berlin (2005).

[15] E. Sangiorgi, B. Ricco and F. Venturi, $\mathrm{MOS}^{2}$ : an efficient Monte Carlo simulator for MOS devices. IEEE Trans. ComputerAided Des. 7 (1988) 259-271.

[16] V. Sverdlov, E. Ungersboeck, H. Kosina and S. Selberherr, Current transport models for nanoscale semiconductor devices. Mater. Sci. Eng. R 58 (2008) 228-270.

[17] R.M. Yorston, Free-flight time generation in the Monte Carlo simulation of carrier transport in semiconductors. J. Comput. Phys. 64 (1986) 177-194. 